\title{
Performance comparison of distributed architectures for content adaptation and delivery of Web resources
}

\author{
Claudia Canali \\ University of Parma \\ claudia@weblab.ing.unimo.it
}

\author{
Valeria Cardellini \\ University of Roma "Tor Vergata" \\ cardellini@ing.uniroma2.it
}

\author{
Riccardo Lancellotti \\ University of Modena \\ lancellotti.riccardo@unimo.it
}

\begin{abstract}
The increasing popularity of heterogeneous Webenabled devices and wired/wireless connections motivates the diffusion of content adaptation services that enrich the traditional Web. Different solutions have been proposed for the deployment of efficient adaptation and delivery services: in this paper we focus on intermediate infrastructures that consist of multiple server nodes. We investigate when it is really convenient to place this distributed infrastructure closer to the clients or to the origin servers, and which is the real gain that can be get by node cooperation. We evaluate the system performance through three prototypes that are placed in a WAN-emulated environment and are subject to two types of workload.
\end{abstract}

\section{Introduction}

The recent proliferation of heterogeneous Web-enabled devices and network connections, and the contemporary growing complexity of Internet-based services have scaled the demand for content adaptation services to an all-time high. The increased penetration of wired and wireless accesses to the Web from highly heterogeneous client devices is now a visible reality. The differences among the present devices (desktops, mobile phones, hand held computers, PDAs, and Web-TVs) concern various resources, such as CPU power, storage capacity, ability to accept and manage data types, and network connectivity. Such an emerging scenario determines the need for solutions which provide efficient adaptation and delivery of heterogeneous Web-based resources. These services are typically obtained through complex operations carried out by intermediary agents that are interposed between the traditional Web client and server processes. These agents may run on the client machines (client-side solutions) or on intermediary server nodes, that may be placed closer to the clients (edge-side solutions) or to the origin servers (origin server-side solutions).
In this paper, we do not consider client-side solutions that may be affected by device technological constraints, and prefer to focus on intermediary distributed architectures. The use of multiple server nodes seems the most suitable approach to build an efficient infrastructure for Web content adaptation and delivery, even because scalability goals require the use of additional functions besides adaptation, such as efficient resource lookup, client request redirections, management of resource replication and caching.

The number of feasible alternative is huge. In this paper we aim to investigate the pros and cons of placing the distributed infrastructure, that executes adaptation services and related functions, closer to the clients or closer to the origin servers. We consider performance results and focus on the impact of WAN effects and workload models, however we are aware that a complete comparison should also include management and cost issues. The reader should be conscious that managing an infrastructure of multiple servers distributed around the world (edge-side solutions) is not comparable to installing and controlling a similar architecture closer to the content provider servers (origin serverside solutions, e.g. [12]). This difference motivates the business market of CDN companies that operate as third parties. These aspects are out of the scope of this paper.

The first proposals of edge-side solutions are represented by multiple stand-alone nodes that are located close to the client devices $[8,11,15]$. An important evolution is represented by architectures that provide some form of cooperation among the nodes of the intermediate architecture $[1,7]$. These cooperative distributed systems, that in this paper are called cooperative edge server-side architectures, are motivated by two factors: the computational cost of adaptation services leads to load sharing; the significant increment of circulating Web resources (original and adapted versions) increases disk space needs and leads to cooperative caching.

The contribution of this paper is to provide the first performance comparison of the three architectures for content adaptation and delivery services that have emerged as leading solutions. Specifically, we consider representative implementations of the origin server-side, the edge server- 
side, and the cooperative edge server-side architectures. The performance evaluation is carried out through experimental prototypes where the number of nodes of the three distributed architectures is kept constant, and a testbed where wide-area network characteristics are reproduced through WAN emulators. This controlled environment allow us to evaluate the sensitivity of the system performance to two main factors: the WAN effects and the workload models. In particular, we carry out a detailed evaluation of the impact of network characteristics such as latency and bandwidth.

Many adaptation services can be carried out in a heterogeneous client environment. In this paper, we focus on image transcoding services because these visual resources still predominate the content of most Web sites. In all the considered architectures, the adaptation services are provided onthe-fly. While this is the only viable solution for the edgeside approach, we have chosen it for the origin server-side architecture because it guarantees the maximum flexibility and generality with respect to off-line counterpart solutions; moreover, it frees the provider from creating in advance and keeping consistent multiple versions of its resources.

There are few experimental performance comparisons of different infrastructures for efficient adaptation and delivery of Web resources. Most of them have been carried out through simulations and aim to demonstrate the improvement of the proposed solutions. After many experiences of experiments of distributed systems in the Internet (e.g., $[1,2])$, we are appreciating the benefits of having a controlled testbed environment where the experiments are scientifically reproducible. For example, the performance evaluation of an origin server-side system for content adaptation provided by Chandra et al. [3] in an uncontrollable real environment does not allow the authors to give a clear picture of the impact of WAN effects. Similar limits characterize performance evaluations of origin server-side architectures [15]. More detailed analyses on WAN effects have been carried out in traditional Web-based services. For example, Dykes et al. [6] provide a detailed analytical model for evaluating the network sensitivity of Web caching.

The investigation of the edge-side architectures, in which the adaptation and delivery services are provided by standalone nodes (or by cluster of nodes [7]), has attracted the attention of many researchers. For example, Han et al. [8] provide an analytical study of edge-side transcoding, but their model uses simplified assumptions on network utilization and does not consider the impact of network congestion. Other studies, such as $[10,11]$, focus on the system architecture and limit their experiments to a LAN scenario. Other performance evaluations of caching in systems for content adaptation have been carried out through simulators [5, 13]. Clearly, the network models in these simulators are characterized by simplifications that cannot take into account most packet-level dynamics and impact of WAN traffic.
Cooperative edge-side architectures for content adaptation have been proposed more recently (e.g., [1, 14]). The study in [14] focuses on content personalization of peer-topeer networks and discusses architectural scalability issues, without taking into account network effects. Other studies [1] on cooperative architectures are based on real uncontrollable networks.

The rest of this paper is organized as follows. Section 2 describes the characteristics of the three intermediary architectures for efficient content adaptation and delivery. Section 3 presents the experimental testbed. Section 4 discusses the experimental results on the performance comparison of the considered architectures. Section 5 concludes the paper.

\section{System architectures}

The basic architecture of a scalable system for efficient content adaptation and delivery services consists of multi-level content adaptation and delivery infrastructure, in which we identify three main levels that is, the client, the origin server and an intermediary level in between the two.

The origin server is the content repository of the original Web resources; specifically, the resources furnished by the origin server may be stored on the disk or generated dynamically through the interaction with some application/database server; in any case, the origin server does not perform any content adaptation operation on them. The intermediary nodes provide content adaptation to transform, when necessary and on the basis of the client context information (i.e., the device characteristics and capabilities), the required resource. For this reason, in the following of this paper we will refer to the intermediary nodes as adaptation servers. The client issues requests for Web resources that are processed by the intermediary; the latter can interact with the origin server to retrieve the original resource before the final delivery to the client.

Since CPU power of a node can be easily exhausted by on-the-fly adaptation services, the resource version resulting from adaptation can be cached, in such a way that subsequent requests for the same version can exploit the possibility of data reuse. However, as there are many device types and adaptation mechanisms, the distributed architecture has to deal with several versions of the same resource.

Due to the presence of multiple versions of the same resource, a multi-version lookup process is necessary which may cause one of the following three events. In case of $e x$ act hit, the cache contains the exact version of the requested resource, which is immediately sent to the client device. If the cache contains a more detailed and adaptable version of the requested resource, a useful hit occurs and the cached object can be transformed to meet the client context information before sending the resource to the client. In case of miss, the cache does not contain any exact or adaptable ver- 
sion of the requested resource and the node must fetch the original resource from the origin server, if necessary adapts it, and then sends the result to the client.

In the following of this section we describe the three architectures compared in the paper. We can distinguish origin server-side adaptation from edge-server side adaptation, whose main difference for the scope of this paper lies in the location of the adaptation servers with respect to the origin servers and the network edge. We further distinguish two types of edge server-side architectures, depending on whether edge servers can cooperate or not among them.

\subsection{Origin server-side architecture}

The origin server-side architecture is operated directly by the content provider for its own resources. In this architecture, adaptation services are carried out directly on the platform of the content provider. Therefore, the adaptation servers are located on the same local network of the origin server and are operated directly by the content provider. As already noted, we consider an origin-server side approach providing on-the-fly adaptation services because of the benefits of this solution with respect to the off-line alternative. However, the drawback of on-the-fly adaptation is intuitive: it may require significant computing power for adaptation services in addition to that of generating and delivering traditional Web-based services. To address the performance issues related to on-the-fly adaptation, the provider platform needs to adopt a distributed architecture with replicated computing resources. In addition, caching of already adapted resources allows to retrieve the requested content from the disk rather than to transform it again. Therefore, we consider a two-tier architecture where the front-end tier consists of multiple adaptation servers acting as enhanced reverse proxies, while the back-end tier is a simple content repository of the original content versions.

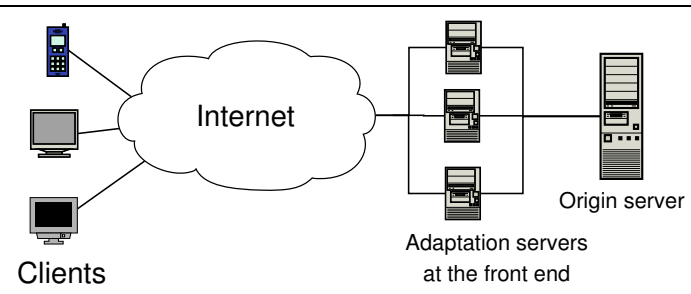

Figure 1. Origin server-side architecture

Fig. 1 illustrates the origin server-side architecture. We can see the the content-provider Web system with the two tiers: the adaptation servers at the front-end level and the origin server in the back-end.

\subsection{Edge server-side architecture}

In the edge server-side architecture, the adaptation servers are located on the network edge close to the clients (the adaptation servers are typically placed within the network of the ISP providing Internet access to the clients). Adaptation services carried out by some edge node seem the most viable solution for the following motivations. First, the large majority of new devices needs a gateway to access Web-based services. Second, edge-based adaptation services can take advantage of caching of already adapted resources saving not only computational power but also network bandwidth. Finally, the adapted content can be served from a node closer to the client than the origin server. Fig. 2 illustrates the edge server-based architecture. The adaptation servers are placed close to the clients and the wide area network is located between these nodes and the origin server.

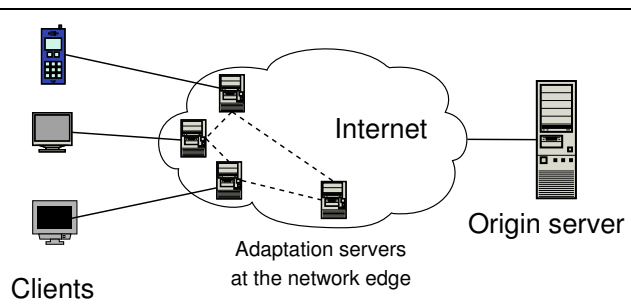

Figure 2. Edge server-side architecture

We can distinguish two classes of edge server-side architectures on the basis of their relationship with the content provider: those operated by a third party company on behalf of its customer content providers and those operated by an independent third party that provides the adaptation and delivery services for all Web resources. Apart the network location of the adaptation servers, the first case does not present many differences with the origin server-side architecture because both solutions limit the adaptation and delivery services to a specific subset of Web resources and can fully exploit a server-directed adaptation process taking into account the semantics of the resource content. On the other hand, the deployment of adaptation services by an independent third party is characterized by a reduced direct interaction with the content provider that does not allow to provide all possible forms of adaptation. However, in this paper we do not consider the provisioning of adaptation services that require a strict interaction with the content provider but we rather consider device-driven adaptation services, where the transformation operations are mainly related to the characteristics of the client device. 


\subsection{Cooperative edge server-side architecture}

The cooperative edge server-side architecture is similar to the edge server-side, with the difference that now the adaptation servers can cooperate. Two forms of cooperation can be exploited in cooperative architectures: the first regards the content location to take advantage of cache contents of nearby nodes, the latter allows the distribution of the content adaptation operations from heavily to lightly loaded nodes. As the cooperative content location has a predominant performance impact [1], in this paper we consider only this form of cooperation. Fig. 2 shows the cooperation as dashed lines connecting the adaptation servers.

The deployment of cooperation for the content location function among the nodes of the intermediate architecture in the context of adaptation services may follow multiple schemes, such as query- or summary-based lookup algorithms. Since in a previous study [1] the authors have compared multiple cooperation schemes and found that a querybased cooperation achieves the best performance thanks to the effectiveness of its resource location algorithm, in this paper we consider a cooperative architecture that uses a query-based cooperation scheme.

When a node receives a client request, it starts a local lookup for the requested resource in the server cache and may require a cooperative lookup process. The query-based scheme performs the cooperative lookup by sending a query message to each peer. A response from a peer causes a remote hit that must be explicitly fetched. When no suitable resource is found in any peer, the requested resource must be fetched from the origin server.

\section{Experimental testbed}

In this section, we describe the workload models and the setup of the experimental environment used to compare the performance of three content adaptation and delivery architectures considered in this paper.

\subsection{Workload models}

We carried out our experiments using two workload models which differ in the nature of the working set. The first model, namely IRcache, aims at capturing a realistic Web scenario with a reduced adaptation load. The set of resources used in this workload is based on proxy traces belonging to the nodes of the IRCache infrastructure. Some characterizations performed on the images of this workload, such as file size, JPEG quality factor, and colors of GIF images, evidence that they are very close to the characteristics reported in [4].

The second workload model aims at denoting a scenario where the adaptation process has a major cost. This work- ing set, namely Photo album, is modeled as a Web photographic album with a significant amount of large pictures. As the trend of the Web is towards a growing demand for graphical and multimedia resources, this workload can represent a likely Web scenario for the future. For this reason, we provide a more detailed discussion of results obtained with this latter workload, using the IRcache workload only for the initial performance comparison.

Client requests are issued to the system using a client emulator according to synthetic traces (in a way similar to the experiments described in [1]). The client request rate is the same for both workloads.

\subsection{System setup}

We set up an experimental test-bench composed of a node acting as a client emulator, 16 nodes equipped with the content adaptation software, and a node with a Web server that acts as the origin server. Each node is a dual-P4 with 1GB of RAM, gigabit Ethernet running Linux 2.6.8.1. Fig. 3 shows the experimental testbed, with the three types of nodes that are used throughout the experiments: client, adaptation servers and origin server. The content adaptation function is provided by a prototype based on Squid [1]. Each adaptation server node manages also a caching space. The cache on each node is configured in such a way to hold $25 \%$ of the global working set for both workloads.

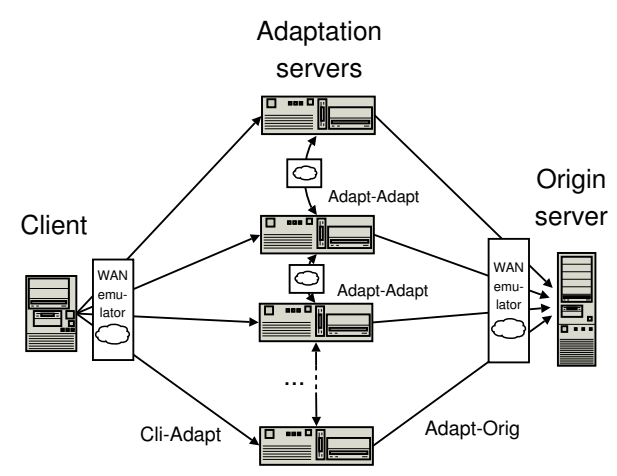

Figure 3. Experimental testbed

To compare the three architectures we introduce WAN emulation on the links connecting the nodes. As shown in Fig. 3, we have three classes of links, namely Cli-Adapt from client to adaptation servers, Adapt-Adapt among adaptation servers, and Adapt-Orig from adaptation servers to origin server. WAN emulation is provided through special packet schedulers that are part of the 2.6 Linux kernel. We simulate three WAN effects: packet loss, packet delay, and bandwidth limitation. Packet loss and delay are provided by the netem packet scheduler, while bandwidth limitation is 
obtained through the token bucket filter traffic shaper. Delay is modeled through a linear combination of Pareto and Gaussian distribution, as suggested in [9].

As the focus of our study is directed towards an architecture comparison at the provider level, we do not take into account the last-mile and we focus on the system part from the client access point to the origin server. In the origin serverside architecture, the adaptation servers are located on the same network of the origin server; therefore, we introduce WAN emulation on Cli-Adapt links. In the edge server-side architecture we introduce WAN effects on the Adapt-Orig links of Fig. 3 that is, the links connecting the adaptation servers to the origin server. Finally, in the cooperative edge server-side architecture we introduce WAN emulation also on the Adapt-Adapt links, in addition to the WAN effects on Adapt-Orig links.

The space of choice of the network emulation parameters is huge, as each link is described by three different parameters (network delay, packet loss probability, and bandwidth). For space reasons, we limit the results of our analysis to a significant subset of the possible combinations. A preliminary study on real networks allowed us to extract a reference set of network emulation parameters. Table 1 shows the network parameters values used in our experiments. The bold values for bandwidth are used in the experiments of Section 4.1, while the bandwidth range is used for the sensitivity analysis to network parameters in Section 4.2.

\section{Experimental results}

In this section we compare the performance of the three considered architectures, using as main index the system response time. However, our analysis takes also into account other indexes, such as cache hit rate and utilization of hardware and operating system resources of the nodes.

We first compare the architectures for the two different workloads to evaluate the sensitivity to the workload characteristics. The two workloads have a different impact on caching performance, computational load, and network resource utilization, hence it is interesting to compare the architectures for two highly different scenarios. In the second set of experiments we use only one workload and we evaluate the performance sensitivity to the network scenario.

\subsection{Sensitivity to workload}

Figs. 4 and 5 show the cumulative distribution of the response time for the origin server- and edge server-side architectures for the IRcache and the Photo album workloads, respectively. A comparison of the two figures shows that the Photo album workload provides higher response times with respect to the IRcache workload. The reason for this performance difference is twofold. First, the Photo

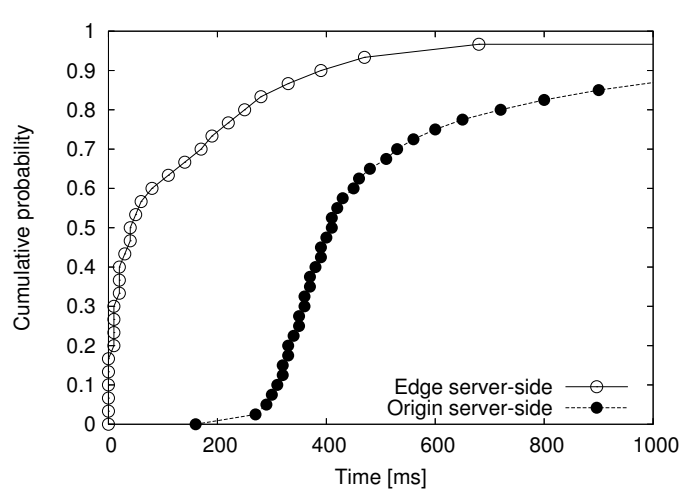

Figure 4. Response time comparison of origin server-side and edge server-side architectures (IRcache workload)

album workload places higher content adaptation requirements than the IRcache workload, leading to an increase in the 90-percentile of adaptation processing time of eight times. Second, resources in the Photo album workload are on average three times larger than in the IRcache workload and this augments the transmission time.

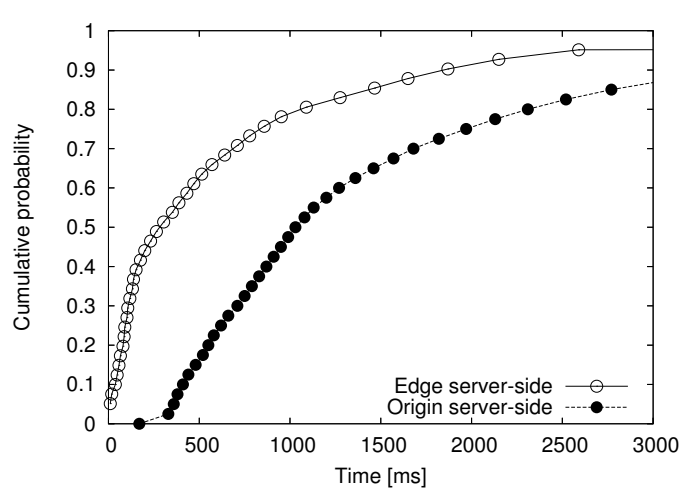

Figure 5. Response time comparison of origin server-side and edge server-side architecture (Photo album workload)

The curves shown in Figs. 4 and 5 demonstrate that the edge server-side architecture provides better performance than the origin server-side architecture for both considered workloads. This performance gain is due to the placement of the adaptation servers close to the clients, that allows the edge server-side architecture to serve client requests resulting in cache hits using only local resources, while WAN links are used only in the case of a cache miss. The hit rate for the edge server-side architecture (which is identical to 


\begin{tabular}{|c|c|c|c|c|}
\hline Architecture & WAN emulated links & Bandwidth [Mbit/s] & Delay [ms] & Loss rate \\
\hline Origin server-side & Cli-Adapt & $8-16-32$ & 100 & $1 \%$ \\
\hline Edge server-side & Adapt-Orig & $8-16-32$ & 100 & $1 \%$ \\
\hline Cooperative edge server-side & Adapt-Orig & $8-16-32$ & 100 & $1 \%$ \\
& Adapt-Adapt & $8-16-32$ & 25 & $1 \%$ \\
\hline
\end{tabular}

Table 1. Reference values for the parameters of the WAN emulator

the hit rate of the origin server-side architecture) is reported in Table 2. From the table we see that the edge-side architecture can serve locally more than $30 \%$ of requests.

\begin{tabular}{|c|c|c|c|c|}
\hline \multicolumn{5}{|c|}{ IRcache workload } \\
\hline Architecture & $\begin{array}{c}\text { Local } \\
\text { exact }\end{array}$ & $\begin{array}{c}\text { Local } \\
\text { useful }\end{array}$ & $\begin{array}{c}\text { Remote } \\
\text { exact }\end{array}$ & $\begin{array}{c}\text { Remote } \\
\text { useful }\end{array}$ \\
\hline Edge server-side & $35 \%$ & $11.3 \%$ & $\mathrm{n} / \mathrm{a}$ & $\mathrm{n} / \mathrm{a}$ \\
\hline Cooperative edge server-side & $31 \%$ & $10.8 \%$ & $9.5 \%$ & $10 \%$ \\
\hline \multicolumn{6}{|c|}{ Photo album workload } \\
\hline Architecture & $\begin{array}{c}\text { Local } \\
\text { exact }\end{array}$ & $\begin{array}{c}\text { Local } \\
\text { useful }\end{array}$ & $\begin{array}{c}\text { Remote } \\
\text { exact }\end{array}$ & $\begin{array}{c}\text { Remote } \\
\text { useful }\end{array}$ \\
\hline Edge server-side & $30 \%$ & $9.5 \%$ & $\mathrm{n} / \mathrm{a}$ & $\mathrm{n} / \mathrm{a}$ \\
\hline Cooperative edge server-side & $25 \%$ & $9.8 \%$ & $15 \%$ & $12 \%$ \\
\hline
\end{tabular}

Table 2. Cache hit rates

If we look at the bottom of graphs in Figs. 4 and 5 (i.e., for cumulative probability below 0.3 ), we see that the origin server-side architecture is characterized by high latency: requests are served in more than $300 \mathrm{~ms}$ and $500 \mathrm{~ms}$ for the IRcache and Photo album workloads, respectively, while for the edge server-side architecture the requests service time is one order of magnitude lower.

We can summarize the results of the comparison between the edge server-side and the origin server-side architecture as follows. For the IRcache workload, we observe that the median response time is reduced by more than ten times and the 90-percentile is reduced by a factor of four. The Photo album workload confirms these results, showing a reduction of four times of the median response time and a 90percentile that has been nearly halved by the edge serverside architecture.

We now focus on the performance improvement achievable by means of lookup cooperation among the adaptation servers. From Table 2 we see that cooperation allows to improve the cache hit rate by nearly $20 \%$, even if perturbations in access locality due to Web resources exchange among the adaptation servers has a slightly detrimental effect on local hit rate with respect to the edge server-side architecture.

Fig. 6 shows the cumulative distribution of the response time for the edge server-side and the cooperative edge server-side architectures for the IRcache workload, while Fig. 7 refers to the Photo album workload. The response times for cumulative probability below 0.3 in Figs. 6 and 7 are mainly related to requests resulting in a local hit. In this case the response times of the two architectures are very

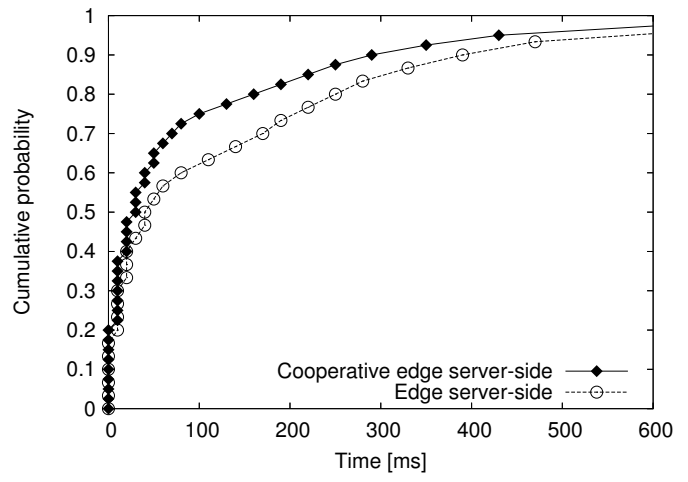

Figure 6. Response time comparison of edge server-side and cooperative edge server-side architectures (IRcache workload)

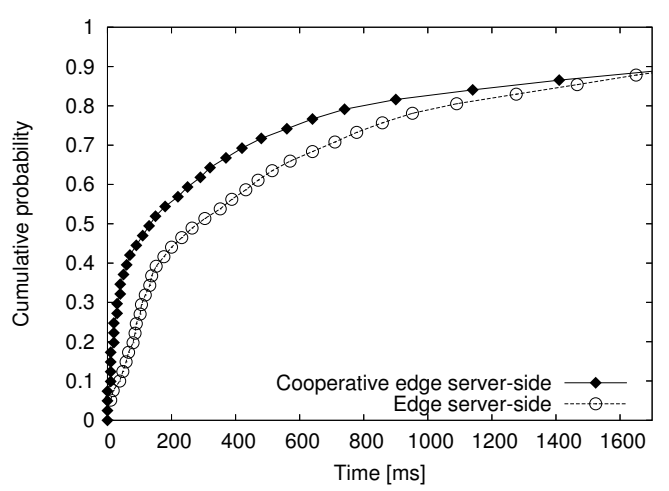

Figure 7. Response time comparison of edge server-side and cooperative edge server-side architectures (Photo album workload)

close. On the other hand, in the case of a local cache miss the cooperative architecture can locate a remote hit on a nearby cache, thus saving both a download from the origin server and, possibly, a content adaptation operation. As in our WAN emulated scenario Adapt-Adapt links are characterized by lower delays with respect to the Adapt-Orig links, remote hits are usually served faster than misses. Hence, if we compare the median response time we can appreciate the performance gain due to cooperation, that is $33 \%$ for the IR- 
cache workload and rises up to $42 \%$ for the Photo album workload. Finally, in the upper region of Figs. 6 and 7, that is related to requests resulting in a miss, the curves come close again because both the architectures handle misses in the same way, thus providing similar response times.

\subsection{Sensitivity to network parameters}

We now evaluate the impact of network parameters on the performance of the considered architectures. We carried out this sensitivity analysis for both workloads; however, due to space reasons, we report the results for the Photo album workload only. Indeed, due to the larger resource size this workload places a heavier load on the network and is therefore more appropriate to analyze the impact of network parameters. Results on the IRcache workload confirms the main findings, but the observed sensitivity to network parameters is less significant. We studied the effect of both network latency and bandwidth limitation in the WAN emulation, but we report only the most interesting results, which are related to the sensitivity analysis to the bandwidth. Indeed, sensitivity to network delay has an impact on response time which is one order of magnitude lower with respect to the sensitivity to network bandwidth.

Table 3 provides a performance comparison of median and 90-percentile of response time of the origin serverside and edge server-side architectures. The response times are referred to values of the bandwidth of the WAN links ranging from 8 to $32 \mathrm{Mbit} / \mathrm{s}$. For both architectures we see clearly the detrimental effect of bandwidth reduction on the performance. However, the most interesting observations arise from the comparison as the bandwidth is reduced. The edge server-side architecture always outperforms the origin server-side architecture and the performance gain augmentes as the bandwidth decreases. For example, the performance gain of the edge-side architecture over the origin server-side approach on the 90-percentile of response time grows from $60 \%$ to $86 \%$ as the bandwidth is reduced from 32 to $16 \mathrm{Mbit} / \mathrm{s}$. Moreover, when the bandwidth is set to $8 \mathrm{Mbit} / \mathrm{s}$ the origin server-side architecture is characterized by a high number of failures (120 seconds, which is the 90-percentile of response time, is the timeout of our client emulator). On the other hand, the edge server-side architecture provides a more graceful performance degradation even in the case of network congestion.

The median response time shown in Fig. 8 as a function of WAN links bandwidth confirms these findings. We observe a growth in performance gain of the edge-side architecture from $417 \%$ to $472 \%$ as the bandwidth is reduced from 32 to $16 \mathrm{Mbit} / \mathrm{s}$. Furthermore, we see clearly the different behavior of the two architectures when the bandwidth is further reduced to $8 \mathrm{Mbit} / \mathrm{s}$.

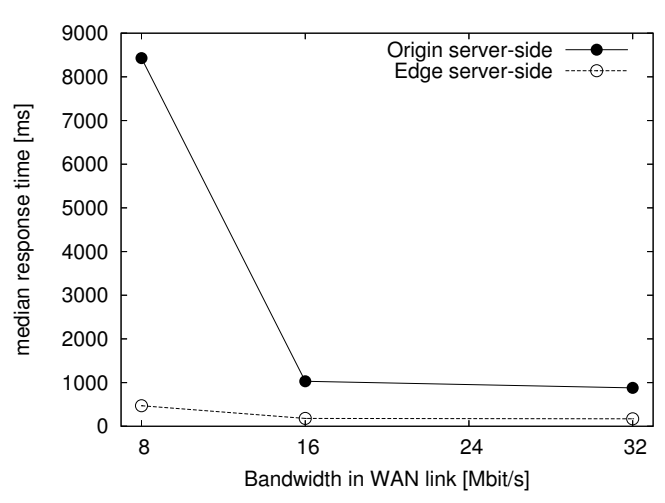

Figure 8. Median response time as a function of the WAN bandwidth

The performance difference between the two architectures has a strong relationship with the number of sockets open during the experiments. Table 3 shows, in the second column, the average number of sockets open on the adaptation servers throughout our experiments as a function of the bandwidth. We observed the number of open sockets to be fairly stable during our experiments, with a standard deviation always below $20 \%$ of the mean value.

\begin{tabular}{|c|c|c|c|}
\hline \multicolumn{4}{|c|}{ Origin server-side architecture } \\
\hline Bandwidth [Mbit/s] & $\begin{array}{c}\text { Average \# } \\
\text { open socket }\end{array}$ & $\begin{array}{c}\text { Response time [ms] } \\
\text { median }\end{array}$ & 90-percentile \\
\hline 8 & 190 & 8430 & 120100 \\
16 & 110 & 1030 & 3440 \\
32 & 40 & 880 & 2620 \\
\hline \multicolumn{3}{|c|}{ Edge server-side architecture } \\
\hline Bandwidth [Mbit/s] & Average \# & \multicolumn{2}{c|}{ Response time [ms] } \\
open socket & median & 90-percentile \\
\hline 8 & 80 & 470 & 54680 \\
32 & 16 & 180 & 1848 \\
& 11 & 170 & 1630 \\
\hline
\end{tabular}

Table 3. Open sockets and response time as a function of the WAN bandwidth

We see from Table 3 that the number of open sockets grows as bandwidth is reduced due to the increase in concurrency in client requests caused by longer service times. If we compare the two architectures we see that the number of open sockets is always less than one third for the edge server-side architecture with respect to the origin serverside approach. This is consistent with the observation that the edge server-side architecture relies on WAN links much less than the origin server-side architecture. As a consequence, the edge server-side architecture results less sensitive to the parameters of the WAN links.

As final sensitivity analysis to network parameters, we 


\begin{tabular}{|c|c|c|}
\hline \multicolumn{3}{|c|}{ Sensitivity to Adapt-Adapt bandwidth } \\
\hline \multirow[t]{2}{*}{ Bandwidth [Mbit/s] } & \multicolumn{2}{|c|}{ Response time [ms] } \\
\hline & median & 90-percentile \\
\hline 8 & 150 & 1960 \\
\hline 16 & 130 & 1870 \\
\hline 32 & 110 & 1790 \\
\hline \multicolumn{3}{|c|}{ Sensitivity to Adapt-Orig bandwidth } \\
\hline \multirow[t]{2}{*}{ Bandwidth [Mbit/s] } & \multicolumn{2}{|c|}{ Response time [ms] } \\
\hline & median & 90-percentile \\
\hline 8 & 170 & 2030 \\
\hline 16 & 130 & 1870 \\
\hline 32 & 110 & 1660 \\
\hline
\end{tabular}

Table 4. Sensitivity to network parameters for the cooperative edge server-side architecture

now evaluate the performance gain obtained by the cooperative edge server-side architecture over the edge server-side architecture and compare these results with the sensitivity analysis of the other architectures. Table 4 shows the median and 90-percentile of response time as a function of the Adapt-Adapt and Adapt-Orig bandwidths.

The results presented in Table 4 show that the introduction of cooperation reduces the sensitivity to the network parameters. In case of local miss, downloads can occur from both neighbor caches or from the origin server. This allows a more fair usage of network resources. Furthermore, when multiple copies of a Web resource are located in different cooperative nodes, the system automatically chooses the fastest responding neighbor, thus contributing to evenly distribute network load across the links.

The final result is that the response time is much more stable than in other architectures even in front of significant bandwidth variations. The maximum performance difference on 90-percentile of response time is only $22 \%$ as bandwidth of both Adapt-Orig and Adapt-Adapt links ranges from 8 to $32 \mathrm{Mbit} / \mathrm{s}$. This is fairly low if we consider that the 90-percentile of response time ranges over at least one order of magnitude for the other architectures. This result is consistent with previous studies of Dykes and Robbins [6], that, focusing on traditional Web caching, have demonstrated that cooperation provides a major benefit in reducing response time variance due to network-related delays.

\subsection{Summary of results}

We can summarize the main findings of our study:

- The edge server-side architecture outperforms the origin server-side architecture for every considered workload and network parameter, with a reduction on the 90-percentile of response time up to three times.

- The performance difference between the edge serverside and the origin server side architectures augments significantly in case of scarce network resources due to the higher sensitivity to network conditions of the origin server-side architecture.

- The introduction of cooperation into the edge serverside architecture provides a further performance gain over the standard non-cooperative edge-side architecture; however, the performance gain of cooperative edge-side architecture over edge server-side architecture is less evident than the performance gain of edge server-side over origin server-side architecture.

- Cooperation in the edge server-side architecture is extremely useful in case of scarce network resources; it has significant effect in reducing sensitivity to network parameters down to almost nothing due to a more efficient utilization of WAN links and thus can avoid network congestion, whereas the other architectures pay high penalties in response time due to network delays.

\section{Conclusions}

In this paper we compared the performance of three architectures for Web content adaptation and delivery services in an reproducible and configurable WAN experimental environment. We found that the placement of content adaptation and caching close to the client devices provides an important performance benefit. We also demonstrated that cooperation between the nodes of an edge server-side architecture improves performance further.

We found that the origin server-side solution is the most sensitive due to the heavy usage of WAN links. The edge server-side architecture reduces the sensitivity to WAN effects thanks to client requests resulting in local hits that can be serviced without involving WAN resources. The cooperative edge server-side architecture is further less sensitive because, in case of local miss, it allows a distribution of network load among multiple edge servers, without needing to contact the origin server for every request that cannot be satisfied locally. As a consequence, the performance of cooperative edge server-side architectures provides the best performance gain in case of scarce network resources.

\section{References}

[1] C. Canali, V. Cardellini, M. Colajanni, R. Lancellotti, and P. S. Yu. Cooperative architectures and algorithms for discovery and transcoding of multi-version content. In Proc. of WCW 2003, Sept. 2003.

[2] V. Cardellini, M. Colajanni, R. Lancellotti, and P. S. Yu. A distributed architecture of edge proxy servers for cooperative transcoding. In Proc. of IEEE WIAPP 2003, June 2003.

[3] C. S. Chandra, S. Ellis and A. Vahdat. Application-level differentiated multimedia Web services using quality aware transcoding. IEEE J. Selected Areas in Comm., 18(12):25442465, Dec. 2000. 
[4] S. Chandra, A. Gehani, C. S. Ellis, and A. Vahdat. Transcoding characteristics of Web images. In Proc. of Multimedia Computing and Networking Conf., Jan. 2001.

[5] C.-Y. Chang and M.-S. Chen. On exploring aggregate effect for efficient cache replacement in transcoding proxies. IEEE Trans. Parallel and Distributed Systems, 14(6):611624, June 2003.

[6] S. Dykes and K. Robbins. A viability analysis of cooperative proxy caching. In Proc. of IEEE Infocom 2001, Apr. 2001.

[7] A. Fox, S. D. Gribble, Y. Chawathe, E. A. Brewer, and P. Gauthier. Cluster-based scalable network services. In Proc. of 16th ACM SOSP, pages 78-91, Oct. 1997.

[8] R. Han, P. Bhagwat, R. LaMaire, T. Mummert, V. Perret, and J. Rubas. Dynamic adaptation in an image transcoding proxy for mobile Web browsing. IEEE Personal Comm., 5(6):817, Dec. 1998.

[9] S. Hemminger. Netem home page. http://developer. osdl. org/shemminger/netem/.

[10] S. Ihde, P. P. Maglio, J. Meyer, and R. Barrett. Intermediarybased transcoding framework. IBM System Journal, 40(1):179-192, 2001.

[11] A. Maheshwari, A. Sharma, K. Ramamritham, and P. Shenoy. TransSquid: Transcoding and caching proxy for heterogeneous e-commerce environments. In Proc. of IEEE RIDE 2002, pages 50-59, Feb. 2002.

[12] R. Mohan, J. R. Smith, and C.-S. Li. Adapting multimedia Internet content for universal access. IEEE Trans. Multimedia, 1(1):104-114, Mar. 1999.

[13] B. Shen, S.-J. Lee, and S. Basu. Caching strategies in trascoding-enabled proxy systems for streaming media distribution networks. IEEE Trans. Multimedia, 6(2):375-386, Apr. 2004.

[14] W. Shi, K. Shah, Y. Mao, and V. Chaudhary. Tuxedo: A peerto-peer caching system. In Proc. of PDPTA 2003, June 2003.

[15] A. Singh, A. Trivedi, K. Ramamritham, and P. Shenoy. PTC: Proxies that transcode and cache in heterogeneous Web client environments. World Wide Web, 7(1):7-28, Jan. 2004. 